\title{
Les valeurs : une question philosophique, un défi pédagogique
}

Values: a Philosophical Question, a Pedagogical Challenge

\section{Eirick Prairat}

\section{OpenEdition}

\section{Journals}

Édition électronique

URL : http://journals.openedition.org/recherchestravaux/1563

DOI : 10.4000/recherchestravaux.1563

ISSN : 1969-6434

Éditeur

UGA Éditions/Université Grenoble Alpes

Édition imprimée

ISBN : 978-2-37747-098-3

ISSN : 0151-1874

Référence électronique

Eirick Prairat, «Les valeurs : une question philosophique, un défi pédagogique », Recherches \& Travaux [En ligne], 94 | 2019, mis en ligne le 20 juin 2019, consulté le 08 septembre 2020. URL : http://

journals.openedition.org/recherchestravaux/1563; DOI : https://doi.org/10.4000/recherchestravaux. 1563

Ce document a été généré automatiquement le 8 septembre 2020

(c) Recherches \& Travaux 


\title{
Les valeurs : une question philosophique, un défi pédagogique
}

\author{
Values: a Philosophical Question, a Pedagogical Challenge
}

\section{Eirick Prairat}

Toutefois j'avoue que j'ai plus trouvé de notions réelles sur l'homme

dans les romanciers et dramaturges, et même dans les poètes. [...] Je ne sais s'il est possible de penser raisonnablement à ses semblables sans cet intermédiaire des fictions. Alain, Éléments de philosophie ${ }^{1}$

1 Dans la première partie de cet article $^{2}$, nous examinons la question des valeurs par le prisme de trois grands débats : la définition de la valeur, la querelle du réalisme et la redécouverte des émotions. Dans la seconde partie, nous montrons que l'enseignement est affaire de valeurs. Que l'on s'interroge sur la valeur de l'enseignement dispensé, sur les valeurs requises pour enseigner ou sur celles que l'école doit transmettre, la valeur est une question omniprésente. Dans la troisième et dernière partie, nous revenons sur la question centrale de toute pédagogie : comment transmettre ? Comment transmettre des valeurs? Nous soulignons le paradoxe qui est au cœur de cette transmission. Nous montrons également que celle-ci requiert, comme en contrepoint, un libre travail d'exploration, affranchi de toute visée transmissive. Et c'est à la littérature notamment qu'incombe cette tâche aussi difficile qu'essentielle.

\section{Une question philosophique}

\section{Comment définir la valeur?}

Qu'est-ce qu'une valeur? C'est une vieille question a-t-on envie de dire, mais c'est assurément le premier débat. Les tentatives les plus récentes essaient de définir l'idée 
de valeur en la rapprochant tout en la distinguant de l'idée de norme. La manière de procéder consiste à confronter un concept à un concept voisin dont on pressent à la fois la proximité et l'éloignement. Toute une série de points soulignent cette proximité distante. La valeur serait plus intimement liée au monde des émotions que ne le serait la norme. Ou encore, contrairement à celle-ci, la valeur admettrait des degrés, on peut en effet être plus ou moins courageux, une situation peut être plus ou moins intéressante. Ou encore le domaine d'application de la valeur serait beaucoup plus ample que celui de la norme qui ne concernerait que le registre de l'action ${ }^{3}$.

Ce débat norme/valeur a fait l'objet de nombreux commentaires car il intéresse l'ensemble des sciences humaines et sociales. Il a également permis de réhabiliter, de manière somme toute assez paradoxale, le concept de norme toujours manipulé avec beaucoup de réticence par les philosophes de l'éducation, qui lui ont très souvent préféré celui de valeur. Or, comme l'écrit justement Patrick Pharo :

Il a existé dans la tradition nietzschéenne et marxiste un usage négatif de l'expression norme sociale qui, loin d'être une source de progrès et de civilisation, exprimait plutôt les préjugés et les intérêts des classes dominantes et faisait obstacle à l'émancipation des opprimés ${ }^{4}$.

Cet usage critique, qui a longtemps prévalu, semble aujourd'hui révolu.

Il faut bien comprendre que ce débat norme/valeur a été une manière de prolonger un autre débat: celui qui, dans le champ de l'éthique normative, opposait et continue d'opposer les tenants d'une morale dite "téléologique " aux tenants d'une morale dite " déontologique ». La première - encore appelée par Sidgwick «morale attractive »en appelle à l'horizon polarisant de la valeur, alors que la seconde - impérative - en appelle à la dimension obligeante du devoir et de la norme ${ }^{5}$. Certains philosophes moraux ont alors tenté de montrer, pour faire prévaloir leur position, que l'on pouvait procéder à une réduction : réduire la valeur à la norme comme l'a notamment suggéré Thomas Scanlon ${ }^{6}$ (1998) ou, à l'inverse, réduire la norme à la valeur comme l'a proposé George-Edward Moore dans ses Principia Ethica ${ }^{7}$. Il semble, sans entrer ici dans le détail de débats éminemment techniques, que ces opérations de réduction se heurtent à de redoutables apories et qu'il est plus pertinent de maintenir distincts, sans trop les disjoindre, les concepts de norme et de valeur.

\section{La nouvelle querelle du réalisme}

5 Rappelons-nous la question qu'adresse Socrate à Euthyphron : «Réfléchis à ce qui suit : est-ce que le pieux est aimé par les dieux parce qu'il est pieux ou est-ce parce qu'il est aimé d'eux qu'il est pieux » (Platon, Euthyphron, 10a). En d'autres termes : est-ce qu'une chose est bonne parce que nous l'aimons (thèse dite subjectiviste) ou est-ce que nous l'aimons parce qu'elle est déjà, d'abord bonne (thèse objectiviste) ? Ce débat n'a cessé de susciter des controverses. Comme le note Ruwen Ogien, «il semble impossible de maintenir une position strictement subjectiviste [...]. Mais il parait tout aussi impossible de maintenir une position strictement objectiviste ${ }^{8} »$. En effet, le subjectivisme assimile la valeur à la préférence, il réduit la valeur aux intérêts ou aux désirs de celui qui apprécie et juge. «Rien n'est bon, rien n'est mauvais, c'est la pensée qui crée le bon et le mauvais » dit Hamlet qui illustre à merveille cette option subjectiviste. 
6 Bernard Williams a montré que l'on peut formuler la thèse subjectiviste de trois manières différentes ${ }^{9}$. On peut l'énoncer sur le terrain ontologique en disant que les valeurs sont subjectives au sens où elles ne correspondent à rien de réel dans le monde. En ce sens, le subjectivisme est un antiréalisme. On peut aussi la libeller sur le terrain épistémologique en disant que les valeurs sont subjectives au sens où elles ne peuvent être dites ni vraies, ni fausses, elles ne sauraient même être justifiées. En ce sens, le subjectivisme est un anti-cognitivisme. On peut enfin formuler la thèse subjectiviste, sur le terrain pratique, en disant que les valeurs sont là uniquement pour exprimer des options personnelles, rien de plus. Le subjectivisme est alors un relativisme. Or, chacun le voit, nous pouvons désirer toute une série de choses qui n'ont guère de valeur, il semble donc périlleux de réduire la valeur à une simple entreprise de valorisation subjective. L'objectivisme, de son côté, tout au moins dans ses expressions les plus affirmées, n'est pas exempt de reproches. Comment penser l'accès à la valeur si celle-ci existe de manière totalement séparée ? Pensons à la beauté. Comment la beauté peutelle exister indépendamment de tout sujet qui la reconnait?

7 Ces débats entre subjectivistes et objectivistes, qui opposaient déjà Platon aux sophistes, ont été récemment réactivés sur le terrain de l'ontologie, c'est ce que l'on a appelé « la querelle contemporaine du réalisme moral ${ }^{10}$ ». Les réalistes affirment que les valeurs sont bel et bien réelles au même titre que les propriétés naturelles, elles appartiennent au «tissu du monde ${ }^{11}$ » ou, pour le dire avec Russell, elles participent à « l'ameublement du monde». Les antiréalistes nient, quant à eux, l'idée que les valeurs puissent avoir une existence ontologique au même titre que les propriétés physiques. Ce débat entre réalistes et antiréalistes donnera notamment naissance à la fameuse théorie de la survenance ${ }^{12}$. Théorie qui consiste à défendre une via media conjurant simultanément les difficultés du subjectivisme (qui affirme que les valeurs ne sont que des valorisations, des projections personnelles, bref qu'elles n'ont pas de réalité propre) et les écueils du naturalisme (qui assimilent purement et simplement les valeurs à de simples propriétés naturelles).

8 La théorie de la survenance affirme, dans sa définition générale, que la valeur, sans pour autant être assimilable à une propriété naturelle des choses, est objective dans la mesure où elle «survient». La valeur vient s'ajouter aux propriétés naturelles, sans être elle-même une propriété naturelle. Ce chêne est grand, il a une écorce brun foncé et un feuillage équilibré, c'est un beau chêne ! La valeur (la beauté en l'occurrence ici) survient sur les propriétés naturelles (la grandeur, la couleur de l'écorce, la forme du feuillage). Elle résulte en quelque sorte des propriétés naturelles sans être elle-même une propriété naturelle. Il y a donc bien une objectivité de la valeur sans que celle-ci appartienne à la réalité du monde. Cette théorie de la survenance a connu de nombreuses déclinaisons. John McDowell ${ }^{13}$ et David Wiggins ${ }^{14}$ proposeront, c'est une des perspectives les plus originales, d'associer la valeur à une qualité seconde. Comme la couleur, elle serait une propriété dispositionnelle, propriété objective qui ne se révèle cependant qu'au moment où elle affecte notre sensibilité.

\section{La redécouverte des émotions}

Une bonne partie de la philosophie morale continentale a défendu, à la suite de Kant, une conception intellectualiste de la morale. D'une phrase: la morale est affaire de raison. La psychologie morale contemporaine d'inspiration piagétienne, de Piaget à 
Kohlberg, ne contredira pas cette approche. Nous assistons aujourd'hui à plusieurs critiques et à plusieurs réhabilitations, à commencer par celle de l'imagination. Longtemps décrite comme "la folle du logis ", elle est aujourd'hui considérée comme une vertu épistémique majeure. Rawls la mentionne comme un «bien premier naturel » au même titre que la santé et l'intelligence ${ }^{15}$ et Nussbaum la fait figurer dans sa liste des dix capabilités nécessaires au bon fonctionnement humain ${ }^{16}$. Les émotions sont elles aussi de retour, liées précisément à la question de la valeur. Comment prenons-nous conscience des valeurs? Comment nous sont-elles révélées? Sur ces questions, les travaux de Christine Tappolet ouvrent de nouvelles perspectives.

10 La philosophe défend l'idée que les émotions (l'admiration, le dégoût, la surprise, la peur) sont des modes d'accès privilégiés aux valeurs. Les émotions joueraient à l'égard des valeurs le même rôle que les expériences perceptuelles à l'égard des formes et des couleurs ${ }^{17}$. Les émotions et les expériences perceptuelles partagent en effet un grand nombre de traits comme leurs propriétés phénoménales, le fait de ne pas être directement soumises à la volonté, le fait d'être appropriées ou encore d'être causées par des réalités environnantes. Ces analogies donnent une grande plausibilité à la thèse selon laquelle les émotions sont des perceptions de valeurs. Or, pour que l'émotion nous révèle une valeur, elle ne doit être ni une pure sensation, ni une simple représentation. D'où cette conception originale défendue par Christine Tappolet, sorte de via media entre les approches qui assimilent l'émotion à une simple sensation (les conceptions dites phénoménalistes) et celles qui réduisent l'émotion à une forme de croyance (les conceptions intellectualistes encore appelées cognitivo-conatives). Expliquons-nous.

11 Les émotions ont certes un contenu mais ce contenu n'est pas d'ordre conceptuel car on peut, par exemple, très bien faire l'expérience de la peur tout en ignorant le concept de danger. Si les émotions ont un contenu et si celui-ci n'est pas de nature propositionnelle, alors elles ne sont ni de simples attitudes réactives (sans contenu) ni des croyances (toujours identifiées à un contenu propositionnel) mais des perceptions. Ces travaux nous invitent à repenser la place des émotions dans le procès éducatif et, plus particulièrement, dans le travail sur les valeurs. N'a-t-on pas fait une place trop belle aux lectures rationalistes? N'a-t-on pas, en formation, accordé trop de place à la justification, au raisonnement et à la délibération? N'a-t-on pas, en somme, oublié de faire une place à la sensibilité et à l'expérimentation? Ces travaux nous invitent, plus fondamentalement, à nous déprendre d'un trop grand intellectualisme et, conséquemment, à ouvrir notre regard à d'autres modalités pédagogiques. C'est à cette question que nous tentons de répondre dans la dernière partie de cet article.

\section{Enseigner : une affaire de valeurs}

\section{La valeur de l'enseignement}

Que vaut l'enseignement dispensé ? À quelle condition a-t-il de la valeur ? Nous devons en convenir : tout enseignement a besoin d'être justifié. Il faut qu'il en «vaille la peine ", comme le dit Reboul ${ }^{18}$. Quel critère le justifie de manière indiscutable? On s'accorde généralement à reconnaître que tout enseignement, dans une société donnée, obéit à une nécessité anthropologique de perpétuation. Perpétuer la société, perpétuer la communauté dans laquelle les nouveaux venus viendront à leur tour s'inscrire pour y 
laisser leur marque. En termes arendtiens, l'enseignement est requis par le souci d'assurer « la continuité de la civilisation constituée ${ }^{19}$ ».

Or, dans les démocraties libérales, où l'individu est reconnu pour lui-même, où il vaut comme sujet singulier, comme fin en soi dirait Kant, la considération anthropologique de perpétuation ne saurait suffire. C'est une condition certes nécessaire mais pas suffisante. L'enseignement ne vaut vraiment la peine que si, par-delà la clause de perpétuation, il est capable de proposer au nouveau venu une catégorie d'activités intrinsèquement valables, c'est-à-dire le conduisant vers un degré supérieur d'accomplissement de lui-même. Disons les choses d'une autre manière: l'enseignement ne vaut vraiment la peine que si, par-delà la garantie de continuation de la société, il sait offrir au nouveau venu les conditions d'une libre autodétermination. Voilà le critère, voilà la valeur qui confère de la valeur à l'enseignement et conséquemment à l'école.

C'est pour cette raison que la réflexion sur la bonne école précède toujours la réflexion sur l'école juste. On doit à nos collègues sociologues d'avoir réactivé le nécessaire débat sur l'école juste. Nécessaire en tant que l'école est une institution publique, car on ne se demande jamais si telle école privée de musique ancienne ou de danse moderne est une école juste, on s'inquiète seulement de savoir si elle est une bonne école. Or l'école, en tant qu'institution qui participe à l'attribution de places et de rôles au sein de la société, doit répondre au réquisit de justice. Mais ce débat n'est pas premier. La réflexion sur la bonne école entendue comme réflexion sur les contenus d'enseignement précède la réflexion sur les critères de justice car les programmes scolaires, comme l'a bien vu Dewey, «incarnent le résultat cumulatif des efforts, des luttes et des succès de la race humaine génération après génération ${ }^{20} »$.

\section{Les valeurs du professeur}

15 Valeur de l'enseignement donné, valeurs du professeur aussi. Nous voudrions ici nous intéresser plus précisément aux valeurs morales du professeur. Trois valeurs sont au cœur de la tâche professorale : la justice, la bienveillance et le tact. Commençons par la valeur première au sens de la plus importante : la justice. Il faut envisager la justice selon deux perspectives distinctes, car le professeur se rapporte à l'élève de deux manières différentes. En tant qu'il se rapporte à un sujet de droits, le maître juste respecte les textes, il n'est pas au-dessus du droit et des lois. Ce n'est pas du formalisme, mais l'assurance donnée à tous qu'ils seront traités de la même manière, dans le respect de leurs prérogatives. Or, l'enseignant ne s'adresse pas seulement à des élèves-sujets de droits ; il s'adresse aussi à des sujets apprenants qui, appréhendés cette fois sous l'angle de leurs capacités, apparaissent très différents les uns des autres. Sujets qui n'ont pas les mêmes motivations, les mêmes désirs d'apprendre et de réussir, sujets qui n'ont pas eu les mêmes chances, les mêmes soutiens familiaux. En tant qu'il s'adresse à des élèves qui sont des sujets apprenants très différents les uns des autres, le maître juste fait vivre la dialectique de l'égalité et de l'inégalité. Égalité dans les exigences, égalité dans les attentes au nom d'une égale perfectibilité. Inégalité en revanche, dans les soutiens, les appuis, les aides ; inégalité dans l'accompagnement au nom de difficultés d'apprentissage bien réelles mais toujours contingentes. La justice magistrale se décline donc selon deux versants : le respect de la légalité en tant que le 
professeur s'adresse à des élèves-sujets de droits, et le souci de l'équité en tant qu'il s'adresse à des élèves apprenants très différents les uns des autres.

Disons quelques mots sur la bienveillance. Beaucoup de choses ont déjà été dites et écrites à son sujet ces derniers temps, et lorsque beaucoup de choses sont dites et écrites alors, inévitablement, quelques bêtises sont aussi dites et écrites. Il n'est pas sérieux par exemple de dire, comme certains ont pu le dire il y a peu de temps, que la bienveillance n'est que de la complaisance ${ }^{21}$. Il ne s'agit pas de plaire mais de veiller, de veiller au bien-être. La bienveillance nous invite à apporter à l'élève-enfant confronté à l'inquiétude, à la désillusion et parfois même, disons-le, à la souffrance, une forme de réconfort.

La troisième valeur est le tact que l'on peut thématiser en le distinguant et en l'opposant à la civilité. Il ne s'agit pas bien évidemment de faire disparaître la civilité, vive la civilité. Mais nous ne pouvons vraiment comprendre ce qu'est le tact qu'en le distinguant de cette autre grande qualité relationnelle qu'est la civilité. La civilité est respect des usages et des conventions alors que le tact se manifeste précisément là où les préconisations et les règles viennent à manquer. Il est à la fois sens de l'adresse et sens de l'à-propos. Sens de l'adresse car quand je parle à un élève, je parle toujours à un élève singulier. Et sens de l'à-propos : sens de ce qui doit être dit et comment cela doit être dit, mais aussi et surtout sens de ce qui doit être tu. Le tact manifeste une sensibilité à autrui où s'esquissent les premiers mots, peut-être d'abord les premiers silences, d'une éthique de la parole. C'est à ce titre qu'il est une valeur essentielle.

\section{Les valeurs à promouvoir}

L'école doit promouvoir des valeurs. À l'évidence des valeurs intellectuelles comme la rigueur, le souci de la preuve ou encore la cohérence logique. Mais doit-elle transmettre des valeurs morales? Est-ce bien sa mission? Et si oui, lesquelles? Sur ce point, le débat est loin d'être consensuel. On peut, me semble-t-il, distinguer trois positions que j'appellerai respectivement abstentionniste, procédurale et substantielle. Explicitons chacune de ces positions. La première - abstentionniste - est celle du libéralisme. Elle consiste à dire qu'en régime libéral, il n'y a ni religion d'État, ni morale d'État. L'État est non seulement tenu à la neutralité religieuse mais il est également soumis à l'exigence de neutralité axiologique. Dès lors, l'école publique n'a pas à proposer d'éducation morale, elle doit s'abstenir un point c'est tout.

La seconde position, que je qualifie de procédurale, est notamment défendue par le camp des instructionnistes et par les disciples d'Habermas toujours enclins à réfuter toute fondation transcendante de la morale. Cette seconde position consiste à dire que les seules valeurs morales qui puissent être transmises sont celles qui sont impliquées dans l'exercice de la discussion, et qu'un enseignement digne de ce nom prend précisément toujours la forme d'une discussion. Discussion et non négociation, car dans une négociation l'accord repose sur l'équilibre des intérêts (et éventuellement sur la neutralisation réciproque des intentions hostiles) alors que dans une authentique discussion l'accord repose sur la libre et commune reconnaissance du meilleur argument. Il n'y a donc pas, dans la perspective procédurale de programmes particuliers à prévoir, même si un inventaire est possible car les valeurs morales à transmettre sont celles impliquées par l'acte d'enseigner, si celui-ci reste fidèle à la 
logique argumentative de la discussion et aux présupposés moraux que celle-ci implique.

La troisième position est la position républicaine. Elle reconnaît un ensemble de valeurs morales précises à transmettre : la liberté, l'égale dignité, la solidarité, la tolérance, le respect, l'esprit de justice... D'où le titre de « substantielle » qui peut lui être également donné. Il faudrait pour être rigoureux parler, comme nous allons le voir, de substantialisme faible. Le raisonnement qui préside à l'établissement de cette position (qui est notamment celle du rapport sur l'enseignement laïque de la morale de 2013) est le suivant. Tout d'abord il reconnaît que l'école républicaine a une mission d'éducation morale car l'idéal républicain ne saurait découler mécaniquement d'un projet d'instruction, aussi complet soit-il. La question majeure est d'inventorier les valeurs à promouvoir, car nos sociétés démocratiques et pluralistes sont marquées par ce que l'on appelle à la suite de Rawls « le fait du pluralisme » (The fact of pluralism), c'est-àdire par l'idée que nos sociétés admettent en leur sein plusieurs conceptions de la vie bonne $^{22}$. Ce pluralisme à propos des conceptions du bonheur n'engage pas seulement chez nos concitoyens des choix existentiels mais également des choix de valeurs ou, tout au moins, une priorisation de certaines valeurs par rapport à d'autres. On peut en effet très bien préférer la prospérité individuelle à l'entraide, les libres rencontres à la fidélité, la sécurité au courage. Il importe alors de délimiter les contours de la morale scolaire pour qu'elle soit compatible avec l'irréductible pluralisme qui caractérise les sociétés contemporaines. «La morale de l'école » ne saurait donc être une morale du Bien, comme elle a pu l'être sous la troisième République, car toute morale du Bien promeut un style de vie particulier auquel nul n'est tenu de souscrire dans une société ouverte et pluraliste. D'où il résulte que la morale à promouvoir ne peut être qu'une morale du juste (ou morale du vivre-ensemble) qui se propose de transmettre les seules valeurs qui engagent notre rapport à autrui au sein d'un espace public, pensé et défini comme lieu de la coexistence des libertés. Ce débat - «L'école doit-elle transmettre des valeurs morales?»- porte à son point d'incandescence un conflit qui, dans le champ scolaire, n'a cessé d'opposer ces dernières décennies libéraux et républicains.

\section{Le défi de la transmission}

\section{Comment transmettre des valeurs?}

Dès lors que l'on accrédite l'idée républicaine qu'il y a des valeurs morales à transmettre se pose la question du «comment». Comment transmettre de telles valeurs ? Ce n'est pas, là encore, un débat nouveau, car Platon dans le Ménon et Aristote dans Éthique à Nicomaque s'étaient déjà demandé si l'on pouvait enseigner la vertu. Comprenons bien que lorsque le professeur enseigne la justice à Paul, ce n'est pas seulement pour lui donner quelques lumières sur l'idée de justice, mais c'est pour qu'il devienne juste. Enseigner des valeurs morales n'est donc pas un enseignement comme les autres, les stratagèmes didactiques les plus sophistiqués ne sauraient suffire. On ne peut esquisser de solution à ce redoutable problème qu'à partir d'une précompréhension de ce que l'on appelle une valeur.

On peut raisonnablement souscrire à l'idée selon laquelle une valeur enferme trois caractéristiques: cognitive, affective et conative. Explicitons chacune de ces caractéristiques. Il y a tout d'abord dans la valeur une dimension cognitive, une idée, 
un contenu pourrions-nous dire pour dire les choses simplement. Si j'explicite la valeur justice, je serai attentif à montrer qu'elle enferme une forme d'égalité qui peut se décliner de deux manières différentes (une déclinaison arithmétique et une déclinaison géométrique). Mais une valeur n'est pas un concept, elle ne saurait se réduire à l'idée qui la définit, elle a aussi une dimension affective. Elle est, comme le mot "valeur " l'indique, ce qui vaut pour moi, ce que je souhaite librement valoriser dans le champ des possibles symboliques. En d'autres termes, je dois pouvoir me dire : «Elle me plaît cette idée de justice et je pressens qu'elle va donner sens à la manière dont je veux m'avancer.» La valeur enferme enfin une dimension conative, elle irrigue nos comportements, elle nous donne des raisons d'agir.

Le paradoxe de la transmission des valeurs morales réside en ce point : il faut enseigner la valeur justice, et bien d'autres encore, aux nouveaux venus mais celles-ci ne peuvent, en raison de leur dimension affective, qu'être librement choisies. La seule manière de sortir de ce cercle (qui consiste à transmettre ce qui ne peut être que librement choisi) est de rendre la valeur désirable. La valeur ne s'impose pas, elle se propose. Elle n'est ultimement choisie que parce que le maître sait la rendre désirable. Il n'y a pas de transmission de valeurs sans exemplarité. Il faudrait prendre le temps de réfléchir à cette idée d'exemplarité, souvent invoquée mais rarement explicitée. Cela étant, les valeurs " passent » aussi par des contenus d'enseignement, elles "passent » enfin et surtout par les modes d'organisation et de régulation institutionnels, par ce que l'on appelle aujourd'hui la forme scolaire. Transmettre des valeurs, ce n'est donc pas seulement donner à les connaître intellectuellement, c'est aussi et surtout les rendre désirables.

\section{Ce qu'il ne faut pas attendre de la littérature}

S'il est une thèse soutenue dans cet article, c'est d'affirmer que tout travail sur les valeurs requiert également, comme en contrepoint, un travail de libre exploration. Nous l'avons souligné dans la section précédente : transmettre des valeurs, ce n'est pas transmettre des savoirs académiques en raison de la structure même de la valeur. Ajoutons que les valeurs sont plurielles et que nulle valeur ne peut prétendre s'imposer à d'autres valeurs de manière logique. Le sociologue Max Weber parlait, il y a près d'un siècle déjà (1919), de "guerre des dieux » pour souligner la pluralité conflictuelle des valeurs dans les sociétés modernes ${ }^{23}$. S'il y a une incommensurabilité des valeurs, si nulle valeur ne peut prétendre être au-dessus d'une autre valeur, alors aucun conflit de valeurs ne peut être résolu de manière froide et rationnelle. Cela exige certes de savoir rendre certaines valeurs désirables, cela exige aussi et surtout d'ouvrir un véritable travail d'exploration. Et c'est cette dernière tâche qui incombe précisément à la littérature.

Ce rapport de la littérature à la question des valeurs et, plus largement, à celle de l'éthique a été examiné par Jacques Bouveresse ${ }^{24}$. En nous inspirant de son propos, nous pouvons présenter trois manières de penser ce rapport. On peut déjà penser, c'est la première manière, il faut l'évoquer même si elle semble aujourd'hui bien désuète, que la littérature a une vocation moralisatrice, qu'elle est là pour nous inculquer des valeurs, pour nous éduquer moralement par son contenu édifiant. L'important dans une telle perspective est le choix du corpus, choix qui doit obéir à une commande morale. Cette première thèse est difficile à soutenir car elle asservit la littérature, elle 
la subordonne à une vulgaire entreprise de moralisation. On peut également penser que la bonne littérature est précisément celle qui échappe ou renonce à ce procès d'édification.

Une seconde manière d'envisager ce rapport est de faire de la littérature une école au service de l'apprentissage. La littérature pour apprendre à raisonner moralement. C'est la position que semble parfois défendre Martha Nussbaum. «Le roman, écrit Nussbaum dans L'Art d'être juste, construit un modèle de style de raisonnement éthique qui est spécifiquement lié au contexte sans être relativiste, et qui nous donne des impératifs concrets susceptibles de devenir des universaux ${ }^{25} »$. Pour comprendre cette tentation, il faut avoir en tête le retour de la casuistique, avoir compris que cette dernière est aujourd'hui en train de renaître de ses cendres ${ }^{26}$. Une décision éthique, pour être juste dans une telle perspective, exige de tenir compte de certaines caractéristiques contextuelles.

La notion de contexte ne doit pas être comprise comme un simple contenant au sein duquel se déploie l'action (arena) mais plutôt comme une configuration (setting) qui est produite, au moins en partie, par l'activité des personnes impliquées au sein de celle-ci. Le contexte n'est donc pas un ensemble fixe de conditions environnantes, mais un processus dynamique lié à l'action même des acteurs. Or la littérature par son souci du concret et du particulier saisit, mieux que tout traité de psychologie, l'épaisseur et la complexité de nos vies. Elle "récupère l'individuel qu'a laissé tomber l'universel en évoquant une émotion, en racontant "une vie"; en même temps (...) elle récupère l'ambigu (...) qui est inhérent à la vie (...) » (Jullien, 2016, p. 19-20). De là à faire de la littérature une didactique au service de la formation morale il n'y a qu'un pas. Mais la littérature n'est pas là pour nous initier à des formes de raisonnement, ce serait à nouveau l'instrumentaliser à des fins qui lui sont extérieures. Si elle participe à cette tâche ce n'est que de manière incidente.

\section{Une place éminente}

28 La troisième et dernière manière d'envisager le rapport littérature/valeurs semble la plus plausible. La littérature augmente notre connaissance morale, non au sens où elle nous apprendrait à raisonner, mais au sens où elle élargit notre perception. Dit autrement, la littérature nous invite à vivre des expériences que nous ne pourrions vivre. «Le romancier n'est-il pas [...], écrit Bouveresse, quelqu'un qui nous invite à participer à des expériences de pensée d'une certaine sorte, dans lesquelles des personnages inventés se trouvent placés dans des situations hypothétiques qui exigent d'eux des décisions qui sont la plupart du temps à la fois difficiles et lourdes de conséquences, et à nous demander avec eux : quelle serait la bonne façon d'agir dans une situation de cette sorte ? Et y en a-t-il seulement une ${ }^{27}$ ?». Le lecteur est convié, ne serait-ce qu'indirectement, à se mettre à la place d'un personnage ou à entrer dans une situation.

Le rapport de la littérature au monde de la valeur n'est donc pas un rapport de formation au sens étroit du terme (moraliser ou apprendre à raisonner), il est plutôt une invitation à expérimenter, à explorer d'autres facettes du réel et à imaginer selon un mode toujours singulier et original de nouveaux possibles. «Si je lis Voyage au bout de la nuit de Céline, écrit Putnam, je n'apprends pas que l'amour n'existe pas, que tous les êtres humains sont odieux et haineux [...]. Ce que j'apprends est à voir le monde 
comme il a l'air d'être pour quelqu'un qui est sûr que cette hypothèse est correcte. Je vois quelle plausibilité a cette hypothèse ; à quoi ressembleraient les choses si elle était vraie [...]. C'est la connaissance d'une possibilité ${ }^{28}$.» Ce n'est que sur fond de possibles qu'un monde peut être librement choisi. Ce n'est que sur fond d'une libre exploration de l'univers des valeurs, que certaines d'entre elles peuvent se détacher pour finalement être élues.

Un dernier point, il tient à l'écriture même. Comme le souligne Avraham B. Yehoshua évoquant L'hôte d'Albert Camus : «[...] La littérature, grâce à la force de sa rhétorique suggestive, parvient à élargir l'horizon de notre univers moral et à l'étendre à des lieux insoupçonnés ${ }^{29}$ ». Élargir notre horizon, pénétrer le sens d'une situation, éprouver l'intensité physique et émotionnelle d'un moment. Nous ne saurions en effet mieux éprouver l'épuisante brutalité de la torture qu'en nous plongeant dans le beau livre de Joseph Andras retraçant la douloureuse histoire de Fernand Iveton, ce militant communiste torturé puis guillotiné lors de la guerre d'Algérie ${ }^{30}$. À l'inverse, la tramwayologie ${ }^{31}$ de Judith Jarvis Thomson et celle, plus récente, du psychologue Joshua Greene, pour importantes qu'elles sont dans leur travail de mise à l'épreuve de nos intuitions morales, ne sont guère de nature à stimuler notre empathie et notre imagination, nous proposant de délibérer froidement sur des dilemmes on ne peut plus abstraits et désincarnés ${ }^{32}$.

\section{NOTES}

1. Alain, Éléments de philosophie [1990], Paris, Gallimard, coll. « Folio essais », 2017, p. 331-332.

2. Précisons que dans cet article nous ne faisons pas de différence entre éthique et morale.

3. R. Ogien et Ch. Tappolet, Les concepts de l'éthique. Faut-il être conséquentialiste ?, Paris, Hermann, coll. «L'avocat du diable», 2008, p.15-28; P. Livet, Les normes, Paris, Armand Colin, coll. « Vocation philosophique », 2006, p. 7-42.

4. P. Pharo, « Normes de culture et normes de raison », dans R. Boudon, P. Demeulenaere, R. Viale (dir.), L'explication des normes sociales, Paris, PUF, coll. « Sociologies », 2001, p. 145.

5. Le philosophe anglais Henri Sidgwick distingue dans les premières pages de The Methods of Ethics (1874) et de Outlines of the History of Ethics (1902) deux grandes conceptions de la moralité : celle qui tend le besoin et le désir de la personne vers quelque chose (la conception attractive) et celle qui l'oblige à faire quelque chose (la conception impérative). Voir H. Sidgwick, Methods of Ethics (1874), London, Macmillan, 1962.

6. T. M. Scanlon, What we Owe to Each Other, Cambridge, Harvard University Press, 1998.

7. G.E. Moore, Principia Ethica, (1903), trad. de M. Gouverneur revue par R. Ogien, Paris, PUF, 1998.

8. R. Ogien, "Normes et valeurs", dans M. Canto-Sperber (dir.), Dictionnaire d'éthique et de philosophie morale, Paris, PUF, 1997 ( $2^{\mathrm{e} e ́ d .), ~ p . ~} 1056$.

9. B. Williams, La fortune morale. Moralité et autres essais, trad.J.Lelaidier, Paris, PUF, coll. «Philosophie morale », 1994, p. 13.

10. S. Virvidakis «La connaissance morale ", dans M. Canto-Sperber (dir.), Dictionnaire d'éthique et de philosophie morale, Paris, PUF, 1996 (2 éd.), p. 304.

11. J.L. Mackie, Ethics, Inventing Right and Wrong, Hardmondsworth, Penguin, 1977, p. 15. 
12. Pour une présentation précise de la théorie de la survenance et de ses nombreuses variantes on peut se reporter à Stélios Virvidakis La robustesse du bien, éd. Jacqueline Chambon, 1996 et à Ruwen Ogien Le réalisme moral, Paris, PUF, 1999.

13. J. Mc Dowell, "Values and secondary qualities», dans T. Honderich (éd.), Morality and Objectivity, Londres, Routledge \& Kegan Paul, 1985, p. 110-129.

14. D. Wiggins, Needs, Values, Truth, Oxford, Blackwell, 1987.

15. J. Rawls, Théorie de la justice, trad. C. Audard, Paris, Seuil, 1997, p. 476.

16. M. Nussbaum, Capabilités. Comment créer les conditions d'un monde plus juste?, trad. S. Chavel, Paris, Flammarion, coll. «Climats», 2012, p.55. Mentionnons deux références récentes et importantes sur la place de l'imagination dans le raisonnement moral: l'ouvrage de Solange Chavel, Se mettre à la place d'autrui. L'imagination morale, PUR, 2011 et, plus récemment, le bel ouvrage de Martin Gibert, L'imagination en morale, Paris, Hermann, 2014.

17. C. Tappolet, Émotions et valeurs, Paris, PUF, coll. « Philosophie morale », 2000, p. 167.

18. O. Reboul, Les valeurs de l'éducation, Paris, PUF, coll. « Premier Cycle », 1992, p. 2.

19. H. Arendt, La Crise de la culture [1972], Paris, Gallimard, coll. « Folio Essais », 1995, p. 122.

20. J. Dewey, L'École et l'enfant, Paris, éd. Fabert, 2004, p. 65.

21. Voir l'article d'Antoine Desjardins publié dans le journal Le Figaro (15 mars 2017). [En ligne] : <http://www.lefigaro.fr/vox/societe/2017/03/15/31003-20170315ARTFIG00294-ecole-quand-labienveillance-devient-complaisance.php>

22. J. Rawls, La justice comme équité. Une reformulation de Théorie de la justice, trad. B. Guillarme. Paris, La Découverte, 2008, p. 58.

23. M. Weber, Le savant et le politique [1919], trad. J. Freund, Paris, 10/18, coll. « Bibliothèque ", 2002.

24. J. Bouveresse, La Connaissance de l'écrivain. Sur la littérature, la vérité \& la vie, Marseille, Agone, coll. « Banc d'essais », 2008.

25. M. Nussbaum, L'Art d'être juste. L'imagination littéraire et la vie publique [1995], trad. S. Chavel, Paris, Flammarion, coll. «Climats», 2015, p. 8. Plus loin elle écrit : «cet aller-retour entre le général et le concret est, à mon avis, inscrit dans la structure du genre, dans la manière de s'adresser à ses lecteurs. De cette manière, le roman construit un paradigme d'un type de raisonnement moral qui est sensible au contexte sans être relativiste, où nous atteignons des prescriptions concrètes potentiellement universalisables en comparant une idée générale de l'épanouissement humain avec une situation concrète [...] » (p. 40). Mais il ne faut pas aller trop vite en besogne car Martha Nussbaum défend dans ce même ouvrage une thèse sensiblement différente, à bien des égards plus pertinente, et que nous présentons dans les dernières lignes de cet article : celle de la libre exploration de possibles. Voir notamment p. 35 : "Mon thème principal est la capacité à imaginer l'effet que cela fait de vivre la vie d'une autre personne qui pourrait, dans d'autres circonstances, être soi-même ou l'un de ses proches. (...). À la différence de la plupart des ouvrages historiques, les œuvres littéraires invitent le plus souvent leurs lecteurs à se mettre à la place de personnes très variées, et à vivre leurs expériences ». Cette thèse est également exposée dans La Connaissance de l'amour.

26. E. Prairat, Les mots pour penser l'éthique, Nancy, PUN - Éditions universitaires de Lorraine, coll. «Questions d'éducation et de formation », 2014, p. 20.

27. J. Bouveresse, La connaissance de l'écrivain, ouvr. cité, p. 115.

28. Putnam cité par Bouveresse, Ibid., p. 59. Une thèse proche est soutenue par plusieurs auteurs dans l'ouvrage coordonné en 2006 par Sandra Laugier Éthique, littérature, vie humaine, Paris, PUF, 2006.

29. A. B. Yehoshua, Comment construire un code moral sur un vieux sac de supermarché. Éthique et littérature, trad. C. Wardi, Paris-Tel Aviv, Éditions de l'éclat, 1999, p. 104.

30. J. Andras, De nos frères blessés, Arles, Actes Sud, 2016. 
31. Allusion au célèbre dilemme du tramway (trolley problem) exposé pour la première fois par Philippa Foot dans, "The Problem of Abortion and the Doctrine of the Double Effect ", Oxford Review, n 5, 1967.

32. Voir J.J. Thomson, « Turning the trolley », Philosophy and public affairs, $n^{\circ} 36,2008$, p. 359-374, et J. Greene, Tribus morales. L'émotion, la raison et tout ce qui nous sépare, trad. S. Kleiman-Lafon, Genève, Éditions Markus Haller, 2017, p. 143-176.

\section{RÉSUMÉS}

Ce texte est structuré en trois parties. Dans la première partie, nous examinons brièvement les grandes interrogations qui retiennent aujourd'hui l'attention des philosophes sur la question des valeurs. Dans la seconde partie, nous montrons que l'enseignement est affaire de valeurs. Que l'on s'interroge sur la valeur de l'enseignement dispensé, sur les valeurs requises pour enseigner ou sur celles que l'école doit transmettre; la valeur est une question omniprésente. Dans la troisième et dernière partie, nous revenons sur la question centrale de tout enseignement: comment transmettre? Comment transmettre des valeurs? Nous soulignons notamment le paradoxe qui est au cœur de cette transmission.

This text is structured in three parts. In the first part, we briefly examine the major questions that today hold the attention of philosophers on the question of values. In the second part, we show that teaching is about values. That one wonders about the value of the education provided, the values required to teach or those that the school must transmit; value is an ubiquitous question. In the third and final part, we return to the central question of all teaching: how to transmit? How to transmit values? In particular, we highlight the paradox that lies at the heart of this transmission.

\section{AUTEUR}

\section{EIRICK PRAIRAT}

Eirick Prairat est Professeur de philosophie de l'éducation à l'université de Lorraine et membre de l'Institut universitaire de France (IUF). Il est également chercheur associé au Groupe de recherche en éthique de l'éducation de l'université du Québec à Montréal (UQAM). Ses travaux actuels portent sur l'éthique de l'enseignement ; il vient de publier chez ESF Sciences Humaines Éduquer avec tact (2017). 